\title{
Customização em massa no processo de provisão de Habitações de Interesse Social: um estudo de caso
}

\author{
Mass customization for social housing project delivery: a \\ case study
}

\section{Jaqueline Taube \\ Ercília Hitomi Hirota}

\author{
Resumo \\ $\mathbf{P}$ \\ esquisas têm demonstrado que os projetos de habitação de interesse \\ social (HIS) desenvolvidos no Brasil não têm atendido às reais \\ necessidades dos usuários, o que acarreta inúmeras adaptações e \\ ampliações, que implicam dispêndio de recursos financeiros, já bastante \\ escassos para essa camada da população, e prejuízos à qualidade da habitação. Este \\ artigo apresenta resultados de uma pesquisa cujo objetivo foi verificar a \\ aplicabilidade da Customização em Massa (CM), utilizada pela indústria de \\ manufatura para melhor atender às demandas dos clientes, no contexto da \\ produção de HIS no PMCMV. A pesquisa consistiu na sistematização da revisão \\ de literatura, buscando uma síntese conceitual sobre CM, e no desenvolvimento de \\ um estudo de caso em companhia de habitação. Este estudo compreendeu uma \\ análise de requisitos de um empreendimento HIS com 2 anos de uso para verificar \\ as características e a diversidade de requisitos não atendidos, e um mapeamento de \\ fluxo de informações, desde o cadastramento de famílias interessadas em HIS \\ (demanda) até a entrega das chaves às famílias contempladas, para identificar \\ barreiras e oportunidades de uso da CM. Os resultados indicam a aplicabilidade da \\ CM segmentada e, com uso de tecnologia pré-fabricada, a possibilidade de \\ inserção de captura de requisitos específicos do cliente antes da entrega das \\ habitações.
}

Palavras-chave: Habitação de interesse social. Customização em massa. PMCMV.

Jaqueline Taube Universidade do Oeste de Santa Catarina São Miguel do Oeste - SC - Brasil

Ercília Hitomi Hirota Universidade Estadual de Londrina Londrina - PR - Brasil

Recebido em 12/01/16 Aceito em 13/01/17

\begin{abstract}
Many researches have shown that social housing projects (SHP) developed in Brazil have not been meeting the users' real needs. As a consequence, many adaptations and extensions have been made that imply spending already scarce funds for this segment of the population. Moreover, very often, it results in poor quality housing. This article presents results of a study which aimed to verify the applicability of Mass Customization (MC), used by the manufacturing industry for being more effective in meeting customer demands, in the context of SHP production in the Minha Casa Minha VIda project (MCMVP). The research consisted of a literature review, searching for a conceptual overview of $M C$, as well as the development of a case study in a housing company. This study comprised a requirements analysis of a two-year SHP company to verify the characteristics and diversity of users' requirements not met, and an information flow map from registering families interested in SHP (demand) to handing over the keys to the families and identifying barriers and opportunities of using $M C$. The results indicate the applicability of the segmented MC and, using prefabricated technology, the possibility of including specific requirements of the client before handing over the keys.

Keywords: Social housing. Mass customization. MCMVP.
\end{abstract}




\section{Introdução}

A adoção de projetos-padrão para empreendimentos habitacionais de interesse social (EHIS) no Brasil, produzidos segundo a lógica da produção em massa, tem resultado na insatisfação dos usuários (BONATTO; MIRON; FORMOSO, 2011; CIUFFOLINI; SHIMBO, 2013; LIMA; FORMOSO; ECHEVESTE, 2011; BRITO; FORMOSO; ECHEVESTE, 2011). Segundo Brandão (2011), as unidades habitacionais entregues não atendem à diversidade de necessidades dos usuários, os quais são levados a adequar as unidades por meio de modificações e ampliações em suas moradias (BRANDÃO, 2011). Em muitos casos, essa tipologia-padrão não é desenvolvida para tais intervenções, o que dificulta a realização das adaptações com qualidade e baixo custo (BRANDÃO, 2011), acarretando prejuízos à qualidade da habitação e ônus ao usuário. Mesmo assim, tais adaptações são apontadas pelos usuários como melhoria de qualidade de vida.

Além dos prejuízos diretos causados aos usuários, essa falta de qualidade dos projetos de HIS também é prejudicial aos programas habitacionais, já que faz com que os usuários não permaneçam em suas moradias, o que acarreta baixa retenção nos imóveis. Bonatto, Miron e Formoso (2011) apontam em seu estudo que o principal motivo pelo qual os usuários não permanecem nas unidades é a inadequação do espaço da habitação, ou seja, a necessidade de mais espaço.

É, portanto, fundamental a busca de alternativas para melhor atender às necessidades dessa população. A Customização em Massa é uma estratégia de negócios que possibilita às empresas fornecedoras de produtos manufaturados e de serviços atender a novas necessidades (FRUTOS; BORENSTEIN, 2003) e, por consequência, aumentar o grau de satisfação dos clientes (BARLOW; OZAKI, 2003). Essa estratégia proporciona aos clientes finais uma gama de opções de produtos, com base em suas necessidades, com custos e tempo de entrega similares ao alcançado na produção em massa (HART, 1995; PINE, 1993).

Sabe-se, no entanto, que o contexto da produção de HIS é complexo e significativamente diferente do da produção manufaturada, mas a literatura já aponta estudos desenvolvidos e relatos de empresas que têm recorrido à $\mathrm{CM}$ para atender a um mercado em que os clientes apresentam uma ampla gama de necessidades (BARLOW et al., 2003; NOGUCHI; FRIEDMAN, 2002; NOGUCHI, 2003; NOGUCHI, 2004a; SHIN et al., 2008).
A carência de áreas urbanas no Japão e as necessidades dos clientes levaram as empresas de construção de habitação, na década de 1990, a fornecer produtos diferenciados (BARLOW et al., 2003). A partir de então, a indústria japonesa tem utilizado a CM na pré-fabricação de habitações para atender a necessidades dos clientes, fazendo uso de componentes modulares produzidos em massa, o que permite redução dos custos, já que os fabricantes mantêm a economia de escala; e permite, ainda, que os clientes possam customizar suas residências de acordo com suas necessidades, com a combinação dos componentes do produto (NOGUCHI; FRIEDMAN, 2002; NOGUCHI, 2003).

É possível transferir essa lógica de desenvolvimento de produto para o contexto da HIS brasileira?

Este artigo apresenta resultados de uma pesquisa que objetivou verificar a aplicabilidade do conceito de CM na provisão de HIS a partir de uma síntese conceitual analítica sobre CM, já que se observou o uso indiscriminado dos termos customização e personalização aplicados ao contexto habitacional, e de um estudo de caso em companhia de habitação no qual foram analisados requisitos de um empreendimento HIS com 2 anos de uso para verificar as características e diversidade de requisitos não atendidos, e um mapeamento de fluxo de informações do processo de seleção e provisão de habitações a famílias com renda de até R\$ 1.600,00, desde o cadastramento de famílias interessadas em HIS (demanda) até a entrega das chaves às famílias contempladas, para identificar barreiras e oportunidades de uso da CM.

\section{Customização em massa}

O termo CM foi primeiramente previsto por Alvin Toffler, em 1970, como uma habilidade tecnológica (PINE, 1993) e foi descrito por Davis (1987' apud DURAY et al., 2000; PINE, 1993; SILVEIRA; BORENSTEIN; FOGLIATTO, 2001) como "[...] uma tendência de produção e distribuição de bens e serviços customizados individualmente para um mercado de massa [...]" (AHLSTROM; WESTBROOK, 1999, p. 262, tradução nossa) em que os produtos poderiam ser fabricados com as especificações dos clientes, como na economia préindustrial; e com o custo de um item produzido em massa (DURAY et al., 2000; SILVEIRA; BORENSTEIN; FOGLIATTO, 2001). 
Como observado, na literatura não há consenso entre os autores a respeito do conceito de $\mathrm{CM}$, pois há aqueles que argumentam que a CM é aplicada a produtos $^{2}$, e outros, a produtos e serviços ${ }^{3}$. Há os que determinam que a customização deva ocorrer ainda durante as fases de design e produção ${ }^{4}$, e outros que estendem a customização para após o recebimento do produto. Por fim, há os que consideram o custo um ponto crucial na $\mathrm{CM}^{5}$, enquanto outros não o relacionam à estratégia de $\mathrm{CM}^{6}$.

Em função disso, Kaplan e Haenlein (2006) delimitam o conceito de CM com base na análise de definições estabelecidas, considerando os seguintes aspectos, conforme a Figura 1:

(a) a CM deve ser aplicada somente aos produtos, e não a serviços ${ }^{7}$;

(b) o cliente deve estar envolvido no processo durante as fases de operação da cadeia de valor do produto $^{8}$, ou seja, exclui a etapa pós-entrega; e

(c) os custos devem ser semelhantes aos dos produtos produzidos em massa.

Essa delimitação concorda com a apresentada por Noguchi (2004a), em que a customização é aquela que acontece antes da entrega do produto, enquanto a personalização é aquela que é feita após a entrega do produto pelo próprio cliente.

Conclui-se então que a CM é uma estratégia de negócios (PINE, 1993) que permite proporcionar aos clientes finais uma gama de opções de produtos com base nas necessidades desses clientes, com custos similares ao alcançado na produção em massa (DAVIS, $1987^{9}$ apud DURAY et al., 2000; NOGUCHI, 2004a; PILLER, 2004; PINE, 1993), com o uso de tecnologias de informação, processos flexíveis de trabalhos (GILMORE; PINE, 1997; PINE; PEPPER; ROGERS, 1995) e estruturas organizacionais flexíveis (HART, 1995).

A CM pode ser abordada segundo duas variações, $\mathrm{CM}$ visionária e $\mathrm{CM}$ prática/funcional, conforme a Figura 2. A primeira delas fornece uma variedade infinita, contemplando todas as exigências dos clientes, e é marcada pelo envolvimento do cliente durante a fase de design. Essa estratégia é voltada para um mercado um a um.

Já a outra ramificação de CM, CM prática/funcional, fornece uma variedade finita, contemplando algumas das opções dos clientes, e é marcada pelo envolvimento do cliente durante as fases de fabricação e montagem. Esta estratégia é voltada a um mercado de alguns indivíduos, mercado segmentado, conforme a Figura 2.

Figura 1 - Onde a CM acontece

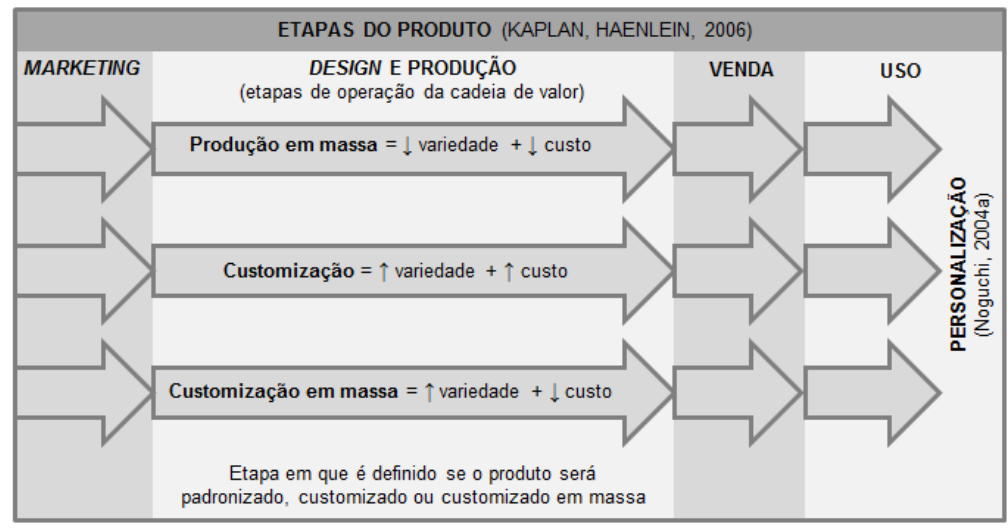

2Segundo Duray et al. (2000), Kumar, Gattoufi e Reisman (2007), Mintzberg (1988* apud Duray et al., 2000), Noguchi (2004a), Piller e Kumar (2006) e Silveira, Borenstein e Fogliatto (2001). *[MINTZBERG, H. Generic Strategies: toward a comprehensive framework. Advances in Strategic Management, v. 5, p. 1-67, 1988.].

${ }^{3}$ Segundo Alford, Sackett e Nelder (2000), Davis (1987* apud AHLSTROM; WESTBROOK, 1999), Gilmore e Pine (1997), Hart (1995), Lampel e Mintzberg (1996), Piller (2004), Piller, Moeslein e Stotko (2004), Pine (1993), Ross (1996), Spira (1996* apud Silveira et al., 2001), Squire (2006) e Tien, Krishnamurthy e Yasar (2004). *[DAVIS, S. M. Future Perfect. Reading: AddisonWesley Publishing, 1987.]. *[SPIRA, Journal of Mass Customization Through Training at Lutron Electronics. Computers in Industry, v. 30, n. 3, p. 171-174, 1996.]

4Segundo Mintzberg (1988* apud Duray et al., 2000) e Noguchi (2004a). *[MINTZBERG, H. Generic Strategies: toward a comprehensive framework. Advances in Strategic Management, v. 5 , p. 1-67, 1988.].

${ }^{5}$ Segundo Duray et al. (2000), Gilmore e Pine (1997), Hart (1995), Kumar (2004), Kumar, Gattoufi e Reisman (2007), Lampel e Mintzberg (1996), Piller, Moeslein e Stokto (2004), Piller e Kumar (2006), Pine (1993), Ross (1996), Silveira, Borenstein e Fogliatto (2001), Squire et al. (2006) e Tien, Krishnamurthy e Yasar (2004).

${ }^{6}$ Segundo Kaplan e Haenlein (2006) e Pine (1993).

${ }^{7}$ Nos serviços, o cliente já está inerentemente envolvido no processo durante a fase de entrega, ou seja, os serviços são sempre customizados (KAPLAN; HAENLEIN, 2006).

${ }^{8}$ As fases de operação da cadeia de valor do produto correspondem às fases de design e produção do produto. ${ }^{9}$ DAVIS, S. M. Future Perfect. Reading: Addison-Wesley Publishing, 1987. 
Figura 2 - Síntese do conceito de CM

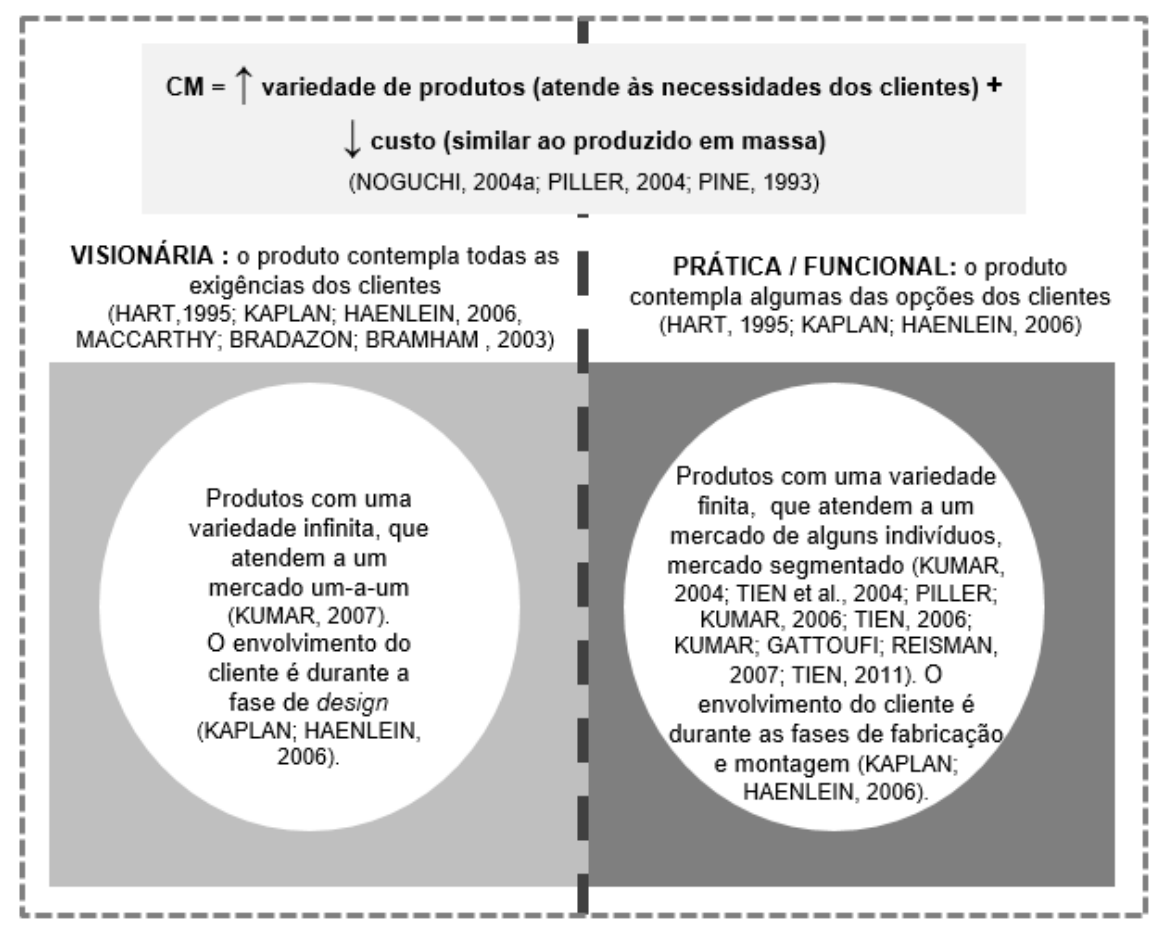

Mesmo após a delimitação da estratégia de CM proposta por Kaplan e Haenlein (2006), um antigo conflito entre visionários e pragmáticos envolvendo o conceito de CM (SILVEIRA; BORENSTEIN; FOGLIATTO, 2001) ainda permanece (KAPLAN; HAENLEIN, 2006) e está diretamente relacionado a quão customizado o produto será, ou seja, ao nível de customização.

Há diversos níveis de customização, ou seja, diversas formas de alcançar a CM, como enfatizam Gilmore e Pine (1997) e Pine (1993). Os níveis de customização são denominados por diversos autores de diversas outras formas: estratégias de $\mathrm{CM}$, estágios da CM, tipos de customização, grau de $\mathrm{CM}$, abordagens de $\mathrm{CM}$, níveis genéricos de $\mathrm{CM}$ e arquétipos de CM. Neste trabalho a nomenclatura utilizada será níveis de customização em massa. Os diferentes níveis de CM foram agrupados com base na nomenclatura estabelecida Silveira, Borenstein e Fogliatto (2001) e correlacionados aos conceitos apresentados neste trabalho, conforme o Quadro 1.

Logo, percebe-se que muitos dos níveis de customização apresentados vão além da CM. Alguns desses níveis referem-se à produção em massa $^{10}$ (padronização, padronização pura, LTO, produção em massa e nível-padrão); outros se referem à personalização ${ }^{11}$ (uso, customização

${ }^{10}$ Segundo definição de Noguchi (2004a).

${ }^{11}$ Segundo definição de Noguchi (2004a).

${ }^{12 S}$ egundo Kaplan e Haenlein (2006), todo serviço já é inerentemente customizado. incorporada, customização feita pelo próprio cliente e adaptativa); e outros, ainda, se referem à customização de serviços ${ }^{12}$ (trabalhos adicionais, serviços adicionais, embalagem e distribuição; ponto de entrega customizado, serviços customizados/resposta rápida, feito sob encomenda, serviços customizados, embalagem customizada, padronização segmentada, customização do produto pós-produção, customização da venda, estética, customização da forma, BTO e customização mínima).

Entre os níveis que se referem à $\mathrm{CM}$, parte deles se refere à $\mathbf{C M}$ prática/funcional ${ }^{13}$ (fabricação, montagem, customização adaptada, customização padronizada, produção modular, montagem modular, ampla variedade de produtos, customização opcional, MTO, ATO montagem sob encomenda e customização parcial). Nesta categoria, a maioria dos níveis descritos possui o ponto de envolvimento do cliente durante a fase de montagem, e só alguns durante a fase de fabricação do produto.

Os demais níveis de CM referem-se à $\mathbf{C M}$ visionária ${ }^{14}$ (fornecedor, design, customização pura, customização do núcleo, colaborativa/transparente, DTO, CMTR, CM e customização completa), na qual a maioria dos

\footnotetext{
${ }^{13}$ Segundo definição de Hart (1995) e de Kaplan e Haenlein (2006).

${ }^{14}$ Segundo definição de Hart (1995), de Kaplan e Haenlein (2006) e de MacCarthy, Bradazon e Bramham (2003).
} 
níveis descritos possui o ponto de envolvimento do cliente durante a fase inicial de design. A definição de personalização em massa dada por Kumar (2007) corresponde à customização pura, pois permite o envolvimento do cliente durante a fase de design, possibilitando uma variedade infinita do produto e o mais alto nível de customização.

Vale ressaltar que, quando um produto é customizado no ponto de entrega, ele não necessariamente é customizado pelo cliente. É o caso de produtos autocustomizados, como um arcondicionado, que é configurado pelo vendedor/instalador de acordo com as necessidades do cliente/ambiente no próprio local de instalação (AHLSTROM; WESTBROOK, 1999).

Como relatado por Barlow et al. (2003) e observado no Quadro 1, a CM pode ser atingida por meio de diferentes níveis. Contudo, as empresas e companhias devem deixar claro quais são os níveis de customização utilizados, assim como o tempo de espera do cliente e os custos resultantes, além de restrições como obrigações contratuais e os fatores relacionados ao processo de desenvolvimento do produto (PDP) e a técnica utilizada, que são intrínsecos a cada nível de customização adotada (BRUN; ZORZINI, 2009).

Duray et al. (2000) verificaram em um estudo empírico que a CM pode ser obtida, na prática, com o uso da modularidade e da identificação estratégica do ponto de envolvimento do cliente no PDP. Esses dois pontos são identificados por Kumar, Gattoufi e Reisman (2007) como os dois componentes principais para a implementação da CM.

A modularidade é importante para a CM, pois é vista como a chave para alcançar a customização a baixo custo (DURAY et al., 2000; PINE, 1993): o design modular do produto proporciona uma ampla variedade de produtos (KUMAR; GATTOUFI; REISMAN, 2007; PILLER; KUMAR, 2006), melhorando, assim, qualidade, custo, flexibilidade, tempo de entrega e serviço das empresas que adotam essa estratégia (KUMAR; GATTOUFI; REISMAN, 2007).

\section{Ponto de envolvimento do cliente}

O ponto de envolvimento do cliente denominado de OPP (Order Penetration Point - OPP) (OLHAGER, 2003) é importante devido à necessidade de o produto ser projetado com as especificações do cliente (DURAY et al., 2000). Dessa forma, a CM atende às necessidades dos clientes por meio da escolha e combinação de componentes padronizados do produto, design modular, feita pelos próprios clientes durante seu envolvimento no PDP (NOGUCHI, 2004a).

\section{Quadro 1 - Níveis de CM $\times$ conceitos}

\begin{tabular}{|c|c|c|c|c|c|c|c|c|c|c|c|c|}
\hline \multicolumn{11}{|c|}{ NIVEEIS DE CUSTOMIZAÇÄO EM MASSA } & \multirow{3}{*}{\multicolumn{2}{|c|}{ Conceitos }} \\
\hline \multirow[b]{2}{*}{$\begin{array}{c}\text { Níveis } \\
\text { genéricos de } \\
\text { CM }\end{array}$} & \multicolumn{10}{|c|}{ Autor(es) / ano } & & \\
\hline & $\begin{array}{c}\text { Mintzberg } \\
\text { (1988 apud } \\
\text { Duray et al., } \\
2000)\end{array}$ & Pine (1993) & $\begin{array}{c}\text { Spira (1996 } \\
\text { apud } \\
\text { SILVIERA; } \\
\text { BORENSTEIN; } \\
\text { FOGLIATTO, } \\
\text { 2001) }\end{array}$ & $\begin{array}{l}\text { Lampel e } \\
\text { Mintzberg } \\
\text { (1996) }\end{array}$ & Ross (1996) & \begin{tabular}{|l} 
Gilmore e \\
Pine (1997)
\end{tabular} & $\begin{array}{c}\text { Alford, } \\
\text { Sackett e } \\
\text { Nelder (2000) }\end{array}$ & \begin{tabular}{|c} 
Piller, \\
Moeslein \\
e Stotko \\
(2004)
\end{tabular} & $\begin{array}{c}\text { Tien, } \\
\text { Krishnamurthy } \\
\text { e Yasar (2004) }\end{array}$ & $\begin{array}{c}\text { Squire et al. } \\
(2006)\end{array}$ & & \\
\hline Fornecedor & & & & & & & & & CMTR & & \multirow{2}{*}{$\begin{array}{c}\text { CM visionária } \\
\text { (HART, 1995; } \\
\text { KAPLAN; } \\
\text { HAENLEIN, 2006; } \\
\text { MACCARTHY; } \\
\text { BRADAZON; } \\
\text { BRAMHAM, 2003) }\end{array}$} & \multirow{4}{*}{$\mathrm{CM}$} \\
\hline Design & $\begin{array}{c}\text { Customização } \\
\text { pura }\end{array}$ & & & $\begin{array}{c}\text { Customização } \\
\text { pura }\end{array}$ & $\begin{array}{c}\text { Customização } \\
\text { do núcleo }\end{array}$ & $\begin{array}{l}\text { Colaborativa / } \\
\text { transparente }\end{array}$ & $\begin{array}{c}\text { Customização } \\
\text { do núcleo }\end{array}$ & DTO & CM & $\begin{array}{c}\text { Customizacãa } \\
\text { completa }\end{array}$ & & \\
\hline Fabricação & $\begin{array}{c}\text { Customização } \\
\text { adaptada }\end{array}$ & & & $\begin{array}{l}\text { Customizacáo } \\
\text { adaptada }\end{array}$ & & & $\begin{array}{c}\text { Customização } \\
\text { opcional }\end{array}$ & мTO & & & \multirow{2}{*}{$\begin{array}{c}\text { CM prática / } \\
\text { funcional (HART, } \\
\text { 1995; KAPLAN; } \\
\text { HAENLEIN, 2006) }\end{array}$} & \\
\hline Montagem & $\begin{array}{c}\text { Customização } \\
\text { padronizada }\end{array}$ & $\begin{array}{l}\text { Produção } \\
\text { modular }\end{array}$ & $\begin{array}{c}\text { Montagem } \\
\text { modular }\end{array}$ & \begin{tabular}{|c} 
Customização \\
padronizada ou \\
padronização \\
customizada
\end{tabular} & \begin{tabular}{|c|} 
Ampla \\
variedade de \\
produtos
\end{tabular} & & & ATO & $\begin{array}{c}\text { Customização } \\
\text { parcial }\end{array}$ & $\begin{array}{c}\text { Customização } \\
\text { parcial }\end{array}$ & & \\
\hline \begin{tabular}{|l} 
Trabalhos \\
adicionais
\end{tabular} & & $\begin{array}{c}\text { Ponto de } \\
\text { entrega } \\
\text { customizado }\end{array}$ & $\begin{array}{l}\text { Feito sob } \\
\text { encomenda }\end{array}$ & & $\begin{array}{c}\text { Customização } \\
\text { do produto pós } \\
\text { produçãao }\end{array}$ & & $\begin{array}{c}\text { Customização } \\
\text { da forma }\end{array}$ & & $\begin{array}{l}\text { Customização } \\
\text { mínima }\end{array}$ & & \multirow{3}{*}{\multicolumn{2}{|c|}{$\begin{array}{c}\text { Serviços } \\
\text { "Customizados", } \\
\text { segundo Kaplan e } \\
\text { Haenlein (2006) todo } \\
\text { serviço já é } \\
\text { inerentemente } \\
\text { customizado }\end{array}$}} \\
\hline $\begin{array}{l}\text { Serviços } \\
\text { adicionais }\end{array}$ & & $\begin{array}{c}\text { Serviços } \\
\text { customizados } \\
\text { e fornecer } \\
\text { resposta } \\
\text { rápida }\end{array}$ & $\begin{array}{c}\text { Serviços } \\
\text { customizados }\end{array}$ & & $\begin{array}{c}\text { Customização } \\
\text { da venda }\end{array}$ & & $\begin{array}{c}\text { Customização } \\
\text { da forma }\end{array}$ & & & & & \\
\hline $\begin{array}{l}\text { Embalagem e } \\
\text { distribuição }\end{array}$ & & & $\begin{array}{l}\text { Embalagem } \\
\text { customizada }\end{array}$ & $\begin{array}{l}\text { Padronizaçâo } \\
\text { segmentada }\end{array}$ & & Estética & & BTO & & & & \\
\hline Uso & & $\begin{array}{l}\text { Customizaçã } \\
\text { o incorporada }\end{array}$ & & & $\begin{array}{c}\text { Customização } \\
\text { feita pelo } \\
\text { próprio cliente }\end{array}$ & Adaptativa & & & & & $\begin{array}{l}\text { Personalizaçãa } \\
\text { (NOGUCH, } 200\end{array}$ & \\
\hline Padronização & & & & $\begin{array}{l}\text { Padronização } \\
\text { pura }\end{array}$ & & & & LTO & $\begin{array}{l}\text { Produção em } \\
\text { massa }\end{array}$ & Nivel padrão & $\begin{array}{l}\text { Produção em ma } \\
\text { (NOGUCHI, 200 }\end{array}$ & $\begin{array}{l}\text { issa } \\
\text { 4a) }\end{array}$ \\
\hline
\end{tabular}


Vale ressaltar que, como abordado por Feitzinger e Lee (1997), para customizar em massa é necessário adiar o Atraso de Diferenciação de Produto (Delayed Product Differentiation - DPD) ou o OPP inicial, para cada cliente, até o último ponto possível na cadeia de produção por meio da integração entre o design do produto e o do processo; e a configuração de toda a rede de abastecimento.

Dessa forma, o produto deve apresentar um design modular, ou seja, o produto é projetado com diferentes módulos, que possibilitam a montagem de diferentes produtos, podendo haver módulos comuns a todos os produtos e outros não, além de um design modular da produção, ou seja, o processo de produção é dividido em módulos, padronizados e customizados a partir do OPP, de modo que eles possam ser reorganizados para abastecer diferentes redes de distribuição de design. A produção deve dispor também de cadeia de abastecimento ágil, de modo que o estoque tenha capacidade e esteja localizado de forma a abastecer a demanda quando necessário. É necessário também que haja integração entre os três, e para isso é necessário que o número de unidades de produção e de distribuição do produto seja compatível (FEITZINGER; LEE, 1997).

Piller e Kumar (2006) complementam dizendo que deve haver a interação entre o cliente e a empresa por meio do co-design. O co-design do produto é entendido como a oportunidade de o cliente interagir com a empresa no momento de criação do produto, ou seja, é o momento em que o cliente configura o produto de acordo com suas necessidades, convertendo-as em especificação do produto (KUMAR, 2007; PILLER; KUMAR, 2006). Essa atividade é responsável pela criação de valor do produto (PILLER, 2004).

O co-design implica, ainda, uma relação direta entre cliente e empresa, o que possibilita a construção de uma relação duradoura, uma vez que o cliente, tendo suas necessidades atendidas, retornará a esse fornecedor, o que possibilita a criação de um banco de dados desses clientes (KUMAR, 2004). No entanto, essa atividade também pode ser exaustiva para os clientes, devido à gama de variedades disponíveis e ao peso das escolhas, o que acarreta, na maioria das vezes, a desistência do cliente (PILLER, 2004; PILLER; KUMAR, 2006).

Uma forma de integrar o processo de design e o processo de produção é considerar OPP ou CODP em ambos os processos (RUDBERG; WIKNER, 2004; WIKNER; RUDBERG, 2005), o que torna possível relacionar as especificações dos clientes a ambos os processos (RUDBERG; WIKNER, 2004). Esta também é uma forma de auxiliar na integração entre o design modular do produto, o design modular do processo e a cadeia de abastecimento, recomendada por Feitzinger e Lee (1997).

\section{Customização em massa na habitação}

A indústria japonesa trouxe a estratégia de CM para o setor habitacional da indústria da construção civil a partir de uma abordagem integradora entre design, produção e comercialização da habitação, denominada de sistema de design customizado em massa (Mass Custom Design System - MCDS) (NOGUCHI; FRIEDMAN, 2002). Segundo esses autores, esse sistema é formulado conceitualmente como: $\mathrm{MCDS}=\mathrm{f}$ (PS).

O sistema de design customizado em massa é dividido em dois subsistemas: o de produtos $(\mathrm{P})$, do qual fazem parte os componentes da habitação, que devem ser modulares-padrão; e o de serviços (S), que envolve a interação entre fornecedores $\mathrm{e}$ usuários. Dessa forma, a MCDS permite uma ampla gama de habitações para seus clientes, atendendo a suas necessidades, com o envolvimento do cliente no PDP durante a escolha dos componentes modulares, que compõem as unidades habitacionais (NOGUCHI; FRIEDMAN, 2002; NOGUCHI; HERNÀNDEZ-VELASCO, 2005).

A escolha da estratégia da CM a ser utilizada pelas empresas implica um processo de tomada de decisões, para o qual Noguchi (2004b) propõe uma sistematização dividida em cinco etapas (Figura 3:

(a) identificação das necessidades: entender a demanda de habitação, ou seja, eliminar as incertezas;

\section{(b) formulação dos objetivos e especificações:} elaborar as especificações;

(c) geração das alternativas: apresentar alternativas que contribuam para CM;

(d) avaliação das alternativas: analisar o valor das alternativas, não só os custos que envolvem o produto, mas também necessidades, desejos e expectativas dos clientes; e

(e) seleção das alternativas: entender os valores reais das alternativas.

As três primeiras etapas são classificadas como fases de CM, enquanto as duas últimas são classificadas como fases de análise de valor, que auxiliam na tomada de decisão final das alternativas de CM mais adequadas, conforme a Figura 3. Portanto, as empresas devem seguir três decisões essenciais: identificação das necessidades; formulação dos objetivos; e especificações e geração das alternativas (NOGUCHI, 2004b). 
Em um estudo empírico, posterior ao apresentado por Noguchi (2004b), Rocha (2011) apresenta dez categorias de decisões que devem ser tomadas na elaboração de estratégias de customização para o setor da construção civil de unidades habitacionais, que podem ser aplicadas a diferentes organizações do setor de habitação (ROCHA; FORMOSO; SANTOS, 2012). Essas dez categorias estão organizadas em quatro categorias macro: central de decisão; arquitetura do produto; interface com o cliente; e operações.

A cadeia de decisões proposta por Rocha (2011) permite que as variações do produto sejam definidas com base nas necessidades dos clientes (ROCHA, 2011; ROCHA; FORMOSO, 2013) e que os problemas associados às escolhas dos clientes para a configuração do produto (PILLER, 2004; PILLER; KUMAR, 2006) sejam eliminados ou evitados (ROCHA; FORMOSO, 2013).

Rocha et al. (2013) argumentam que o uso da estratégia de customização aumenta o número de informações a ser gerenciadas para atender às necessidades dos clientes; e que, se o nível de customização for alto, as configurações dos produtos feitas pelos clientes devem ocorrer off-line com a ajuda de designers, enquanto, se o nível de customização for baixo, as configurações dos produtos feitas pelos clientes podem ocorrer on-line e sem ajuda.
Tillmann e Formoso (2008) identificaram quatro táticas de customização passíveis de ser utilizadas na produção habitacional: customização sob medida; padronização customizada; customização por trabalhos adicionais; e configuração tardia. Essa nomenclatura foi adaptada da literatura para que melhor caracterizasse as táticas.

Contudo, somente dois dos níveis apresentados por Tillmann e Formoso (2008), customização sob medida e padronização customizada, correspondem a níveis de CM. Os outros dois níveis, customização por trabalhos adicionais e customização tardia, correspondem a serviços "customizados" e personalização, conforme o Quadro 2.

Sabendo que os diferentes níveis de customização correspondem às diversas formas para alcançar a CM (GILMORE; PINE, 1997; PINE, 1993) e que a identificação do OPP e da modularidade do produto são fundamentais para determinar o nível de CM do produto (DURAY et al., 2000), esses três pontos compõem os pontos nevrálgicos para a implementação da CM.

Portanto, neste trabalho são considerados como níveis de $\mathrm{CM}$ aqueles que se restringem à estratégia de CM, conforme o Quadro 3, a seguir, desconsiderando os níveis que se referem à padronização, à personalização e a serviços "customizados", conforme apresentado anteriormente, no Quadro 1.

Figura 3 - 0 delineamento do modelo de escolha da estratégia de CM para habitação

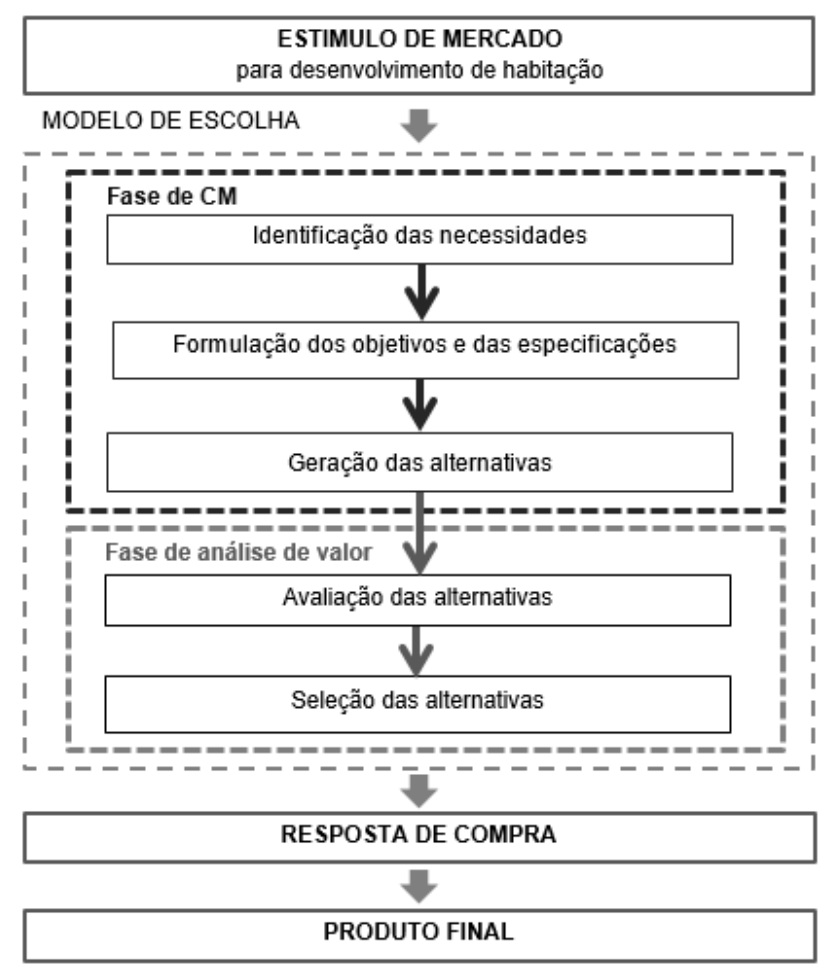

Fonte: adaptado de Noguchi (2004b, p. 18). 
Quadro 2 - Comparação entre os níveis

\begin{tabular}{|c|c|c|c|}
\hline $\begin{array}{c}\text { Silveira et al. } \\
\text { (2001) }\end{array}$ & $\begin{array}{c}\text { Tillmann e Formoso } \\
\text { (2008) }\end{array}$ & \multicolumn{2}{|c|}{ Conceitos } \\
\hline Design & $\begin{array}{c}\text { Customização sob } \\
\text { medida }\end{array}$ & $\begin{array}{c}\text { CM visionária (HART, 1995; } \\
\text { KAPLAN; HAENLEIN, 2006; } \\
\text { MACCARTHY; BRADAZON; } \\
\text { BRAMHAM, 2003) }\end{array}$ & $\begin{array}{c}\text { CM } \\
\text { (vários } \\
\text { autores) }\end{array}$ \\
\hline Montagem & $\begin{array}{c}\text { Padronização } \\
\text { customizada }\end{array}$ & $\begin{array}{c}\text { CM prática / funcional } \\
\text { (HART, 1995; KAPLAN; } \\
\text { HAENLEIN, 2006) }\end{array}$ & \\
\hline $\begin{array}{c}\text { Trabalhos } \\
\text { adicionais }\end{array}$ & $\begin{array}{c}\text { Customização por } \\
\text { trabalhos adicionais }\end{array}$ & $\begin{array}{c}\text { Serviços "customizados". Segundo Kaplan e } \\
\text { Haenlein (2006) todo serviço já é } \\
\text { inerentemente customizado }\end{array}$ \\
\hline Uso & Customização tardia & Personalização (NOGUCHI, 2004a) \\
\hline
\end{tabular}

Quadro 3 - Síntese dos níveis de CM $\mathrm{x}$ denominações de CM

\begin{tabular}{|c|c|c|}
\hline \multicolumn{3}{|l|}{ Níveis de CM } \\
\hline Denominações e Autor(es) / ano & \multicolumn{2}{|c|}{ Conceitos } \\
\hline CMTR (TIEN; KRISHNAMURTHU; YASAR, 2004) & \multirow[b]{2}{*}{$\begin{array}{l}\text { CM visionária } \\
\text { (HART, 1995; } \\
\text { KAPLAN; } \\
\text { HAENLEIN, } \\
\text { 2006; } \\
\text { MACCARTHY; } \\
\text { BRADAZON; } \\
\text { BRAMHAM, } \\
\text { 2003) }\end{array}$} & \multirow{4}{*}{$\begin{array}{c}\text { CM (vários } \\
\text { autores) }\end{array}$} \\
\hline $\begin{array}{l}\text { Colaborativa ( GILMORE; PINE, 1997); CM (TIEN; } \\
\text { KRISHNAMURTHU; YASAR, 2004); Customização do núcleo } \\
\text { (ALFORD; SACKETT; NELDER, 2000; ROSS, 1996); Customização } \\
\text { completa (SQUIRE et al., , 2006); Customização pura (LAMPEL; } \\
\text { MINTZBERG, 1996; MINTZBERG, 1988 apud DURAY et al. , 2000); } \\
\text { Customização sob medida (TILLMANN; FORMOSO, 2008); Design } \\
\text { (SILVIERA; BORENSTEIN; FOGLIATTO, 2001); DTO (PILLER; } \\
\text { MOESLEIN; STOTKO, 2004) }\end{array}$ & & \\
\hline $\begin{array}{l}\text { Customização adaptada (LAMPEL; MINTZBERG, 1996; MINTZBERG, } \\
1988 \text { apud DURAY et al. , 2000), Customização opcional (ALFORD; } \\
\text { SACKETT; NELDER, 2000); Fabricação (SILVIERA; BORENSTEIN; } \\
\text { FOGLIATTO, 2001); MTO (PILLER; MOESLEIN; STOTKO, 2004) }\end{array}$ & \multirow{2}{*}{$\begin{array}{l}\text { CM prática / } \\
\text { funcional } \\
\text { (HART, 1995; } \\
\text { KAPLAN; } \\
\text { HAENLEIN, } \\
\text { 2006) }\end{array}$} & \\
\hline $\begin{array}{l}\text { Ampla variedade de produtos (ROSS, 1996); ATO (PILLER; } \\
\text { MOESLEIN; STOTKO, 2004); Customização padronizada (LAMPEL; } \\
\text { MINTZBERG, 1996; MINTZBERG, } 1988 \text { apud DURAY et al. , 2000; } \\
\text { TILLMANN; FORMOSO, 2008); Customização parcial (TIEN; } \\
\text { KRISHNAMURTHU; YASAR, 2004; SQUIRE et al. , 2006); Montagem } \\
\text { (SILVIERA; BORENSTEIN; FOGLIATTO, 2001); Montagem modular } \\
\text { (SPIRA, 1996); Produção modular (PINE, 1993) }\end{array}$ & & \\
\hline
\end{tabular}

Por fim, outros aspectos importantes devem ser considerados na adoção da CM. Primeiramente, as empresas devem ser claras sobre os níveis de customização do produto, o tempo de espera do cliente e os custos adotados por elas (BARLOW; OZAKI, 2003).
Em seguida, deve haver comunicação entre as equipes/fases de marketing, design e produção apontados por Hart (1995). Portanto, o marketing deve coletar as informações corretamente e também reduzir os intermediários entre o cliente e a empresa; o design deve entender as necessidades dos clientes, e as informações devem ser 
transformadas em especificações dos clientes de forma rápida, flexível e suportável pelo cliente; o cliente deve ter o mínimo de conhecimento da ferramenta utilizada para a coleta das necessidades dele; e a produção deve receber as informações dos clientes no momento necessário à produção (HART, 1995).

\section{Método de pesquisa}

Este artigo apresenta resultados de uma pesquisa cujo objeto foi o processo de seleção e provisão de HIS. A estratégia de pesquisa adotada foi o estudo de caso, que, segundo Yin (2005), é adequado quando se analisa um fenômeno contemporâneo em que se busca analisar, de forma detalhada e em profundidade, um fenômeno dentro de seu contexto e sobre o qual o pesquisador tem pouco ou nenhum controle. Essa estratégia permite lidar com uma ampla variedade de evidências, como artefatos, entrevistas e observações. Foi desenvolvido um estudo de caso único, no Departamento de Assistência Social, setor responsável pelo envolvimento com o cliente final, desde o cadastro até a provisão de HIS, da Companhia de Habitação em uma cidade de médio porte (cerca de 550 mil habitantes).

O trabalho foi desenvolvido em três etapas. A primeira consistiu na revisão bibliográfica, realizada ao longo de toda a pesquisa, com o intuito de criar uma base teórica para auxiliar no desenvolvimento da pesquisa, cuja síntese é apresentada neste artigo.

A segunda e a terceira etapa fazem parte de um estudo de caso único em que o fenômeno analisado foi o processo de provisão de HIS e que foi motivado pela oportunidade de desenvolvê-lo em parceria com a companhia responsável pela entrega do maior empreendimento habitacional do PMCMV à época do desenvolvimento desta dissertação de mestrado da autora deste artigo. No município em que foi realizado o estudo há uma demanda por habitações para atender cerca de 15.500 famílias com renda até 1,5 salário mínimo (COHABLD/2013). A segunda etapa consistiu na análise de requisitos de um empreendimento HIS, com 2 anos de uso, seguida pelo mapeamento de fluxo de informações no processo de seleção e provisão de habitações a famílias com renda de até

\footnotetext{
${ }^{15}$ Esses questionários foram aplicados "face a face", portanto, conforme Zeisel (2006* apud IMAI, 2007, p. 159), "[...] pode ser considerado como uma entrevista, ainda que geralmente as entrevistas tenham um caráter mais aberto, enquanto que os questionários buscam obter a opinião dos usuários por meio de respostas do tipo sim/não ou múltipla escolha [...]". *[ZEISEL, J. Inquiry by Design: tolls for environment-behavior research. New York: Cambridge University Press, 2006.]

${ }^{16}$ Entrevista estruturada é aquela em que o entrevistador segue um roteiro programado, semelhante a um questionário, e as
}

R\$ 1.600,00, desde o cadastramento de famílias interessadas em HIS (demanda) até a entrega das chaves às famílias contempladas (terceira etapa).

$\mathrm{Na}$ segunda etapa, o objetivo foi verificar as características e a diversidade de requisitos não atendidos dos usuários do empreendimento em análise. Para tanto foram identificadas as opções de customização requeridas pelos clientes do PMCMV, por meio da base de dados coletados em uma APO realizada por um grupo de pesquisa entre os meses de janeiro e março de 2013 no empreendimento em estudo. Essa base de dados é considerada como dados secundários neste estudo, já que a APO foi desenvolvida por uma equipe de pesquisadores (CONCEIÇÃO; IMAI; URBANO, 2015). O empreendimento é composto de 1.272 unidades habitacionais unifamiliares e de 1.440 unidades habitacionais multifamiliares, o que totaliza 2.712 unidades habitacionais. Nessa APO foram aplicados questionários ${ }^{15} \mathrm{com}$ moradores de uma amostra de 93 unidades habitacionais unifamiliares, considerando-se o intervalo de confiança de $95,5 \%$ e a margem de erro de $10 \%$, valores estes frequentemente utilizados em pesquisas sobre ambiente e comportamento (ORNSTEIN; ROMÉRO, 1992). O questionário foi baseado no modelo utilizado por Imai (2007).

Entre os dados coletados na APO, foram selecionadas para compor os dados secundários oito questões, sendo quatro questões fechadas (três dicotômicas e uma de múltipla escolha), duas questões semiabertas e duas questões abertas. Nas questões fechadas de múltipla escolha havia um conjunto de opções preestabelecidas para a escolha do respondente, buscando expressar a opinião dele por meio de uma escala de valor (RHEINGANTZ et al., 2009).

$\mathrm{Na}$ terceira etapa, o objetivo foi identificar barreiras e oportunidades de uso da CM no processo de provisão de HIS. Para isso o mapeamento foi dividido em quatro partes paralelas. A primeira consistiu em quatro entrevistas, a primeira estruturada ${ }^{16} \mathrm{e}$ as demais semiestruturadas, ${ }^{17} \mathrm{com}$ o responsável pelo contato com os clientes e coleta de documentos no Departamento de Assistência Social da Cohab. O objetivo dessa parte consistiu em identificar a possibilidade de inserção de pontos de envolvimento do cliente no processo de provisão de

respostas são livres dentro de uma conversação guiada pelo roteiro, diferentemente dos questionários (RHEINGANTZ et al. 2009).

${ }^{17}$ Entrevista semiestruturada é aquela em que o entrevistador segue um roteiro ou esquema básico composto de um conjunto de perguntas que não precisam ser aplicadas sequencialmente (RHEINGANTZ et al., 2009). 
HIS em Londrina-PR na faixa de renda de $\mathrm{R} \$$ $1.600,00$, identificar e compreender como ocorre o processo de provisão e identificar quais são as informações coletadas sobre candidatos ao longo do processo, os critérios de seleção utilizados no munícipio e as diretrizes estabelecidas para o processo de seleção.

$\mathrm{Na}$ segunda parte foram realizadas duas entrevistas semiestruturadas, com um engenheiro do Setor Técnico de Engenharia e Arquitetura da Cohab e uma arquiteta da Gidur da SR da CEF. O objetivo foi o de identificar interfaces desses setores no processo de provisão da HIS, esclarecer informações referentes às análises de viabilidade e de projetos dos EHIS obtidas na primeira etapa e, assim, complementar a compreensão do processo como um todo.

Já na terceira parte, foram obtidas informações junto a um empreendedor responsável pelo desenvolvimento de HIS em Londrina-PR, por meio de correspondência eletrônica, na qual foi enviado um questionário ${ }^{18}$ com questões abertas. Além do questionário respondido o empreendedor também enviou documentos utilizados durante o processo. As informações coletadas correspondem a informações específicas, do fluxo do processo de provisão, referentes ao PDP de EHIS, que envolve desde as etapas iniciais de análises de viabilidade até a entrega dos de EHIS. O objetivo desta parte consistiu em identificar interfaces do agente empreendedor com o processo de provisão da HIS, esclarecer informações obtidas nas etapas anteriores e, desta forma, complementar o mapeamento de atividades e a compreensão do processo de provisão na faixa de renda estudada.

$\mathrm{Na}$ quarta parte foram realizadas as análises de todos os dados coletados nas entrevistas, no questionário e nos documentos fornecidos, gerando um mapa de fluxo organizacional, do processo de provisão de HIS. O empreendimento analisado para elaboração do mapeamento do fluxo do processo de provisão corresponde a um empreendimento diferente do analisado na APO. Por fim, foi feita a análise do fluxo do processo de seleção e provisão da HIS, visando estruturar o processo de provisão de HIS e identificar barreiras e oportunidades para customizar em massa na HIS destinadas a famílias com renda de até $\mathrm{R} \$ 1.600,00$.

O mapa do processo de provisão de HIS foi elaborado em várias etapas, correspondentes às revisões, detalhamentos e validação do mapa, desenvolvidos com a consulta aos entrevistados nas etapas anteriores ou conforme as informações eram coletadas e analisadas. Para a representação do fluxo foi adotada uma sequência de símbolos recomendados por Damelio (2011) para o mapeamento do processo. Todas essas etapas de elaboração do mapa ocorreram com o intuito de complementar e detalhar o fluxo.

\section{Resultados e discussão}

A análise dos dados coletados na APO (segunda etapa da pesquisa) apontou que a CM é viável nesse contexto, pois as modificações realizadas e pretendidas pelos clientes poderiam ser contempladas durante o processo de desenvolvimento das UHs por meio da CM.

Dos dados coletados foram analisadas as seguintes variáveis:

(a) número de unidades que sofreram modificações, quantidade; $\mathrm{e}$

(b) tipos de modificações feitas por unidade.

Entre as modificações feitas as alternativas eram ampliação de cômodos, criação de novos cômodos, adaptação de cômodos e outras modificações. Cada uma dessas quatro opções apresenta uma gama de intervenções realizadas nas UHs; e, ainda, essas opções poderiam ocorrer independentemente umas das outras, conforme apresentado na Tabela 1.

Além das modificações realizadas, foram analisadas as modificações pretendidas pelos entrevistados, a quantidade de ambientes das UHs e o que o entrevistado faria de diferente se pudesse construir sua própria casa. Esses dados demonstram que seis das sete primeiras modificações pretendidas concentram-se na criação de novos cômodos, na ampliação dos cômodos e da casa, e na organização do leiaute da UH, além da opção de murar o lote, identificada entre as sete primeiras opções. Como mencionado anteriormente, as primeiras modificações concentram-se na criação de novos cômodos e na ampliação dos cômodos e da UH, talvez impulsionadas pela insatisfação com a quantidade de ambientes da $\mathrm{UH}$, que $57 \%$ dos respondentes avaliam como ruim ou péssima e que somente $8 \%$ avaliam como ótima.

a presença do pesquisador [...]” (RHEINGANTZ et al., 2009, p.

79).
${ }^{18}$ Questionário é “[...] um instrumento de pesquisa que contém uma série de perguntas relacionadas com um determinado assunto ou problema, que devem ser respondidas por escrito sem 
Tabela 1 - Tipos de modificações feitas por UHs

\begin{tabular}{|c|c|c|}
\hline Tipos de modificações feitas por unidade & no de UH's & $\%$ de UH's \\
\hline Outros & 61 & $69,32 \%$ \\
\hline Criação de novos cômodos e outros & 19 & $21,59 \%$ \\
\hline Adaptação de cômodos e outros & 2 & $2,27 \%$ \\
\hline Criação de novos cômodos & 1 & $1,14 \%$ \\
\hline Criação de novos cômodos e adaptação de cômodos & 1 & $1,14 \%$ \\
\hline Ampliação de cômodos, adaptação de cômodos e outros & 1 & $1,14 \%$ \\
\hline Ampliação de cômodos, criação de novos cômodos, adaptação de cômodos e outros & 1 & $1,14 \%$ \\
\hline Criação de novos cômodos, adaptação de cômodos e outros & 1 & $1,14 \%$ \\
\hline Ampliação de cômodos e outros & 1 & $1,14 \%$ \\
\hline Total & 88 & $100,00 \%$ \\
\hline
\end{tabular}

A necessidade de ampliação, a criação de novos cômodos, a separação entre sala e cozinha, e a adaptação de alguns cômodos caracterizam diferentes possibilidades de leiaute das UHs. Essas modificações estão presentes tanto nos dados levantados acerca das modificações executadas e pretendidas pelos clientes quanto no que o cliente faria de diferente se ele pudesse construir sua própria habitação. Essas características poderiam ser identificadas em um ponto do processo que permitisse selecionar um tipo de projeto (no caso de oferta de projetos padronizados por segmento): são pontos importantes para a definição do programa de necessidades, organização e configuração das UHs. Outra característica importante na consideração do tipo de projeto é a tipologia da $\mathrm{UH}$, em que $18 \%$, aproximadamente, dos entrevistados identificaram que não fariam a casa geminada se pudessem construir sua própria habitação.

Outras demandas como execução de muro e de calçada, alteração do revestimento de piso e parede, instalação de portão, box e gabinete para o banheiro, e execução de horta/jardim poderiam ser identificadas e consideradas em outro momento do PDP porque não interferem no programa da habitação.

Portanto, a análise permitiu, também, identificar que as informações referentes a esses requisitos demandados pelos clientes devem ser consideradas em diferentes pontos do processo, e, por consequência, as diversas informações dos clientes devem alimentar o PDP em diversos pontos do processo.

Ainda, há requisitos que deveriam ser identificados e contemplados em um ponto inicial do processo, o que permitiria selecionar um tipo de projeto, já que eles estão relacionados a pontos importantes para a definição do projeto das UHs, tais como definição do programa de necessidades, organização, configuração e tipologia das UHs. Há, também, os requisitos que deveriam ser identificados e contemplados em um ponto mais avançado do processo porque não interferem no projeto na $\mathrm{UH}$.
O mapeamento do processo de provisão de HIS, resultado da terceira etapa da pesquisa, partiu da premissa de que a demanda por habitações pode ser segmentada de acordo com as características da família, conforme foi demonstrado por Conceição (2015). Dessa forma, a primeira oportunidade para customizar em massa ocorre por meio da identificação e da segmentação das famílias a partir da consulta à base de dados disponível na Companhia de Habitação, composta de informações das famílias que buscam por habitação. No caso estudado, a base de dados disponível poderia conter informações mais específicas dos requisitos das famílias, de forma a possibilitar maior detalhamento na segmentação. Além disso, essa base de dados deve estar atualizada, e deve ser verificado se não há idiossincrasias de informações, conforme apresentado na Figura 4.

Outra oportunidade observada é a adoção do design modular para viabilizar a CM das UHs, o que possibilitaria a organização e a configuração das unidades com base nos segmentos identificados na base de dados. Por meio da seleção prévia das famílias, que ocorre na fase 8 do processo, é indicado que sejam selecionados $10 \%$ (conforme a Portaria $n^{\circ} 595$ ) a mais do número de famílias que serão comtempladas com as UHs. O número reduzido de famílias previamente selecionadas para ser contempladas indica também uma oportunidade de CM, pois reduz a margem de erro da identificação das necessidades das famílias contempladas e, por consequência, facilita essa identificação, conforme apresentado na Figura 4.

Há a possibilidade de envolver o cliente no PDP em um processo de $\mathrm{CM}$ segmentada para inserção de suas necessidades nas especificações do produto por meio de uma melhor sincronização entre o processo de seleção das famílias e a execução das obras se for utilizada uma tecnologia mais rápida, como a pré-fabricada, com montagem de painéis ou de módulos. As informações dos clientes (OPPs) entram no processo analisado na fase 1 , em que ele manifesta interesse em ser contemplado com uma 
UH, e os primeiros dados dessas famílias são levantados; na fase 7, quando um empreendimento é lançado, os clientes interessados manifestam o interesse por ele, e os dados dos clientes são levantados novamente, conforme apresentado na Figura 4.

Figura 4 - Síntese do mapeamento do fluxo do processo de provisão de HIS

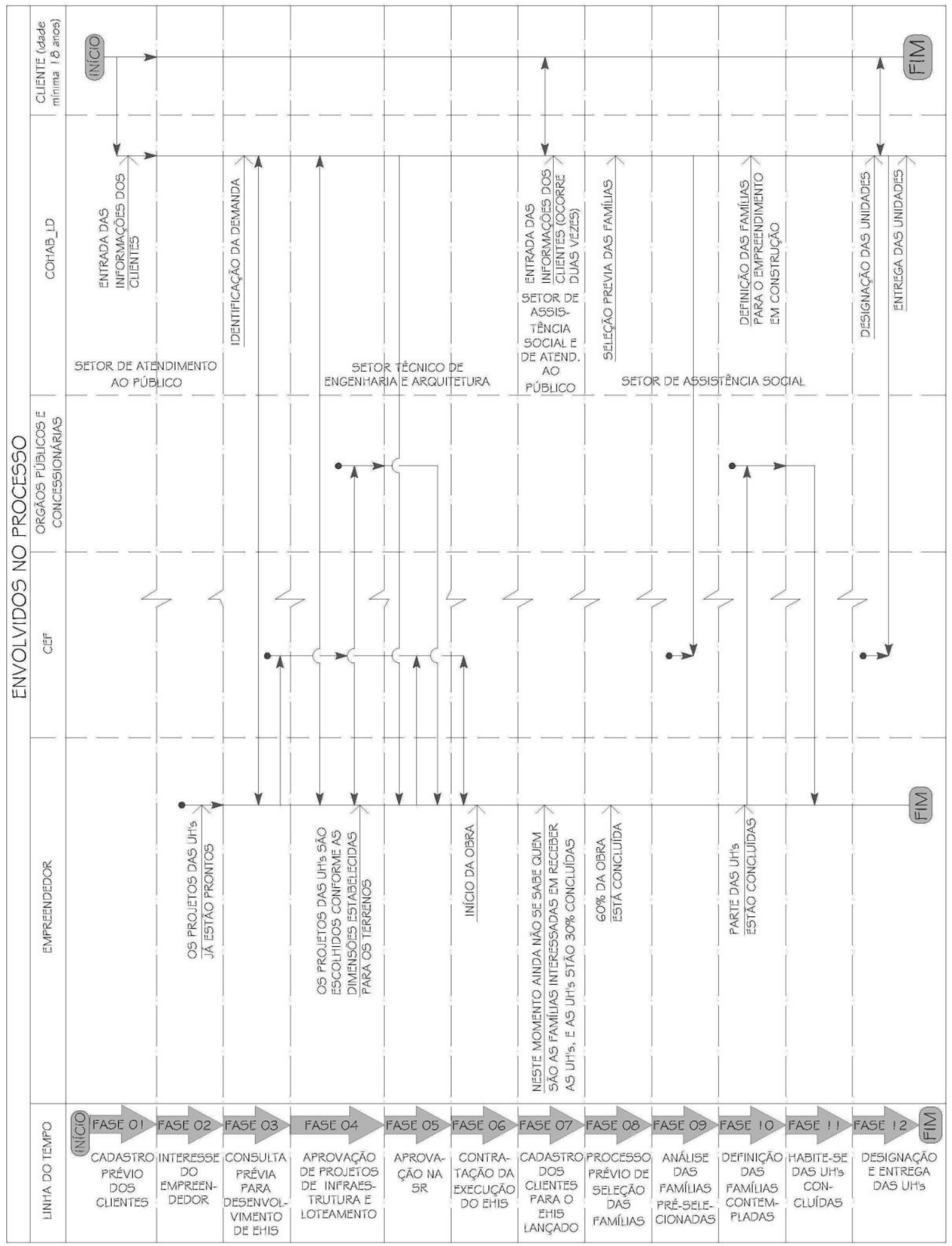


Dessa forma, seria possível capturar as necessidades dos clientes no início do PDP e transferi-las para o produto ao longo de todo o processo, de forma a proporcionar o atendimento dessas necessidades e, consequentemente, maior satisfação do cliente.

As barreiras atuais identificadas para a CM são:

(a) as necessidades dos clientes não são capturadas na etapa de cadastro de famílias interessadas (demanda);

(b) os projetos das UHs são desenvolvidos sem considerar as necessidades dos clientes;

(c) a tecnologia adotada nos EHIS é a tradicional, o que acarreta longo período de execução e, por isso, a obra precisa ser iniciada antes da seleção das famílias, tendo como consequência a não consideração das necessidades dos clientes no PDP; e

(d) a definição das famílias (aprovação da concessão de financiamento) e a designação das UHs a essas famílias só ocorrem após a conclusão das unidades, o que inviabiliza, mais uma vez, a consideração de necessidades específicas da família no processo de desenvolvimento do produto e aumenta o risco de baixa retenção das famílias, conforme já apontado por Bonatto, Miron e Formoso (2011).

Para aumentar a permanência das famílias nas unidades habitacionais, considera-se importante aumentar a eficácia no atendimento às necessidades dessas famílias, o que implica abordar, de forma simultânea e convergente, os dois processos: de desenvolvimento do produto, incluindo a produção das habitações; e de seleção das famílias.

No que se refere ao desenvolvimento do produto, um dos aspectos a ser considerado é a tecnologia construtiva adotada: a tecnologia tradicional para a produção habitacional em larga escala demanda longo período de produção e implica a gestão de grande variedade de recursos. Essas duas características impõem sérias restrições à designação de unidades habitacionais específicas a famílias devidamente identificadas.

Entretanto, se o sistema construtivo permitisse maior agilidade e flexibilidade, o OPP do cliente, ou a definição das famílias, poderia ser próximo à fase final das unidades.

\section{Conclusão}

A CM pode ser uma maneira de agregar mais valor ao produto para o cliente final e, dessa forma, obter maior retenção das unidades. A interação entre os dados coletados do cliente e o ponto de envolvimento com o processo são cruciais para implementar a CM, pois proporcionam o conhecimento das necessidades de cada cliente e o momento em que elas são inseridas no PDP, desde que o processo esteja preparado para isso.

Os resultados obtidos na pesquisa desenvolvida indicam que há barreiras e oportunidades para CM nas HIS destinadas a famílias com renda de até R\$ 1.600,00. Foram identificadas opções de customização requeridas para a faixa de renda mensal de até R $\$ 1.600,00$ de HIS, realizada por meio da análise da base de dados coletados em uma APO.

A partir do mapeamento, foi identificada a possibilidade de inserção de pontos de envolvimento do cliente no processo de provisão de HIS em Londrina-PR nessa faixa de renda, nas fases 1 e 7 .

Por fim, acredita-se que a combinação da estratégia de CM segmentada com uso de tecnologia préfabricada deve possibilitar a inserção de captura de requisitos específicos do cliente.

Nesse sentido, podem ser desenvolvidas pesquisas sobre ferramentas de análise e segmentação das famílias que demandam por habitação, considerando o perfil das famílias e os requisitos demandados por elas; formas para facilitar e sistematizar a transformação dos requisitos dos clientes em atributos do produto; possibilidades de unidades de customização e de espaço de soluções possíveis de ser implementadas, buscando atender a um número maior de necessidades dos clientes.

\section{Referências}

AHLSTROM, P.; WESTBROOK, R. Implications of Mass Customization for Operations Management. International Journal of Operations \& Production Management, v. 19, n. 3, p. 262-274, 1999.

ALFORD, D.; SACKETT, P.; NELDER, G. Mass Customization: an automotive perspective.

International Journal Production Economics, v. 65, p. 99-110, 2000.

BARLOW, J. et al. Choice and Delivery in Housebuilding Lessons From Japan fo UK Housebuilders. Building Research \& Information, v. 31, n. 2, p. 134-145, 2003.

BARLOW, J.; OZAKI, R. Achieving 'Customer Focus' in Private Housebuilding: current practice and lessons from other industries. Housing Studies, v. 18, n. 1, p. 87-101, 2003. 
BONATTO, F. S.; MIRON, L. I. G.; FORMOSO, C. T. Avaliação de Empreendimentos Habitacionais de Interesse Social Com baSe na Hierarquia de Valor Percebido Pelo Usuário. Ambiente Construído, Porto Alegre, v. 11, n. 1, p. 67-83, jan./mar. 2011.

BRANDÃO, D. Q. Disposições Técnicas e Diretrizes Para Projeto de Habitações Sociais Evolutivas. Ambiente Construído, Porto Alegre, v. 11, n. 2, p. 73-96, abr./jun. 2011.

BRITO, J. N. D. S.; FORMOSO, C. T.; ECHEVESTE, M. E. Análise de Dados de Reclamações em Empreendimentos Habitacionais de Interesse Social: estudo no Programa de Arrendamento Residencial. Ambiente Construído, Porto Alegre, v. 11, n. 4, p. 151-166, out./dez. 2011.

BRUN, A.; ZORZINI, M. Evaluation of Product Customization Strategies Through Modularization and Postponement. International Journal Production Economics, v. 120, p. 205-220, 2009.

CIUFFOLINI, M. A.; SHIMBO, L. Z. Políticas Urbanas e Habitacionais e Seus Efeitos Sociais: um estudodo Programa "Minha Casa Minha Vida" no Brasil e na Argentina. In: BONELLI, M. D. G.; LANDA, M. D. V. (Orgs.). Sociologia e Mudança Social no Brasil e na Argentina. São Carlos: Compacta, 2013.

COHAB/LD. PMH - Plano Municipal de Habitação: Gestão 2013-2016. Londrina, Paraná, 2013. Disponível em:

<http://www1.londrina.pr.gov.br/dados/images/sto ries/Storage/cohab/regularizacao_fund>. Acesso em: 7 maio 2014.

CONCEIÇÃO, P. A. Método Para Classificação de Famílias Visando à Adoção da Customização em Massa Por Segmentos na Habitação de Interesse Social. Londrina, 2015. $166 \mathrm{f}$. Dissertação (Mestrado em Arquitetura e Urbanismo) - Universidade Estadual de Londrina, Londrina, 2015.

CONCEIÇÃO, P. A.; IMAI, C.; URBANO, M. R. Captura e Hierarquização de Requisitos do Cliente de Habitação de Interesse Social a Partir da Avaliação Pós-Ocupação e da Técnica de Preferência Declarada. Gestão \& Tecnologia de Projetos, v. 10, n. 1, p. 79-98, 2015.

DAMELIO, R. The Basic of Process Mapping. $2^{\text {nd }}$. ed. New York: CRC Press, 2011.

DURAY, R. et al. Approaches to Mass Customization: configurations and empirical validation. Journal of Operations Management, v. 18, p. $605-625,2000$.
FEITZINGER, E.; LEE, H. L. Mass Customization at Hewlett-Packard: the power of postponement. Harvard Business Review, v. 75, n. 1, p. 116121, 1997.

FRUTOS, J. D.; BORENSTEIN, D. ObjectOriented Model for Custumer-Building Company Interaction in Mass Customization Enviroment. Journal of Construction Engineering and Management, v. 129, n. 3, p. 302-313, 2003.

GILMORE, J. H.; PINE, B. J. The Four Faces of Mass Customization. Harvard Business Review, v. 75, n. 1, p. 91-97, 1997.

HART, C. W. L. Mass Customization: conceptual underpinnings, oppotunities and limits. International Journal of Services Industry Management, v. 6, n. 2, p. 36-45, 1995.

IMAI, C. A Utilização de Modelos Tridimensionais Físicos em Projetos de Habitação Social: o projeto casa fácil. São Paulo, 343 f. Tese (Doutorado em Arquitetura e Urbanismo) - Faculdade de Arquitetura e Urbanismo, Universidade de São Paulo, São Paulo, 2007.

KAPLAN, A. M.; HAENLEIN, M. Toward a Parsimonius Definition of Traditional and Eletronic Mass Customization. The Journal of Product Innovation Management, v. 23, p. 168182, 2006.

KUMAR, A. Mass Customization: metrics and modularity. The International Journal of Flexible Manufacturing Systems, v. 16, p. 287 311, 2004.

KUMAR, A. From Mass Customization to Mass Personalization: a strategic transfomation.

International Journal of Flexible

Manufacturing Systems, v. 19, p. 533-547, 2007.

KUMAR, A.; GATTOUFI, S.; REISMAN, A. Mass Customization Research: trends, directions, diffusion intensity, and taxonomic frameworks. International Journal of Flexible Manufacturing System, v. 19, p. 637-665, 2007.

LAMPEL, J.; MINTZBERG, H. Customizing Customization. Sloan Management Review, v. 38, p. 21-30, 1996.

LIMA, L. P.; FORMOSO, C. T.; ECHEVESTE, M. E. S. Proposta de Um Protocolo Para o Processamento de Requisitos do Cliente em Empreendimento Habitacionais de Interesse Social. Ambiente Construído, Porto Alegre, v. 11, n. 2, p. 21-37, abr./jun. 2011. 
MACCARTHY, B.; BRADAZON, P. G.; BRAMHAM, J. Fundamental Modes of Operation for mass. International Journal of Production Economics, v. 85, p. 289-304, 2003.

NOGUCHI, M. The Effect of the Quality-Oriented Production Approach on the Delivery of Prefabricated Homes in Japan. Journal of Housing and the Built Enviroment. v. 18, p. 353-364, 2003.

NOGUCHI, M.; FRIEDMAN, A. A Design System Approach to Mass Customizing Industrialized Homes in Japan. Technology and Housing, Portland, n. 10-13, p. 114-121, 2002.

NOGUCHI, M.; HERNÀNDEZ-VELASCO, C. R. A 'Mass Custom Design' Approach to Upgrading Conventional Housing Development in México. Habitat International, v. 29, p. 325-336, 2005.

NOGUCHI, M. A Choice Model for Mass Customization of Lower-Cost and HigherPerformance Housing in Sustainable Development. Montreal, 2004. 367 f. Thesi (Doctor of Philosophy in Architecture) - School of Architecture, McGill University, Montreal, 2004a.

NOGUCHI, M. A Proposed Choice Model for the Delivery of Mass Custom Homes. In: INTERNATIONAL HOUSING RESEARCH CONFERENCE, Toronto, 2004. Proceedings... Toronto: CUCS, 2004b.

OLHAGER, J. Strategic Positioning of the Order Penetration Point. International Journal Production Economics, v. 85, n. 3, p. 319-329, 2003.

ORNSTEIN, S. W.; ROMÉRO, M. Avaliação Pós-Ocupação do Ambiente Construído. São Paulo: Studio Nobel, 1992.

PILLER, F. T. Mass Customization: reflections on the state of the concept. The International Journal of Flexible Manufacturing Systems, v. 16, p. 313-334, 2004.

PILLER, F.; KUMAR, A. For Each, Their Own: the strategic imperative of mass customization. Industrial Engineer, p. 40-45, 2006.

PILLER, F. T.; MOESLEIN, K.; STOTKO, C. M. Does Mass Customization Pay?: an economic approach to evaluate customer integration. Production Planning \& Control, v. 15, n. 4, p. 435-444, jun. 2004.

PINE, B. J. Mass Customizing Products and Services. Strategy \& Leadership, v. 21, n. 4, p. 655, 1993.

PINE, B. J.; PEPPER, D.; ROGERS, M. Do You Want to Keep Your Customers Forever? Harvard Business Review, p. 103-114, 1995.
RHEINGANTZ, P. et al. Observando a Qualidade do Lugar: procedimentos para a avaliação pós-ocupação. Rio de Janeiro: UFRJ, 2009.

ROCHA, C. G. da. A Conceptual Framework for Defining Customisation Strategies in the HouseBuilding Sector. Porto Alegre, 2011. 222 f. Tese (Doutorado em Engenharia Civil) - Escola de Engenharia, Universidade Federal do Rio Grande do Sul, Porto Alegre, 2011.

ROCHA, C. G. da; FORMOSO, C. T. Configuring Product Variants in Customization Strategies for House-Building. Open House International, v. 38, n. 3, p. 48-56, 2013.

ROCHA, C. G. da; FORMOSO, C. T.; SANTOS, A. dos. An Overview the Customisation Strategies Developed by Four Organisations of HouseBuilding Sector. In: ANNUAL CONFERENCE OF THE INTERNATIONAL GROUP FOR LEAN CONSTRUCTION, 20., San Diego, 2012. Proceedings... San Diego: IGLC, 2012.

ROCHA, C. G. da et al. Managing the Information Flow in Customized apartment building projects. In: ANNUAL CONFERENCE OF THE INTERNATIONAL GROUP FOR LEAN CONSTRUCTION, 21., Fortaleza, 2013. Proceedings... Fortaleza: IGLC, 2013.

ROSS, A. Mass Customization-Selling Uniqueness. Manufacturing Engineer, v. 76, n. 6, p. 260-263, 1996.

RUDBERG, M.; WIKNER, J. Mass Customization in Terms of the Customer Order Decoupling Point. Production Planning \& Control, v. 15, n. 4, p. 445-458, 2004.

SHIN, Y. et al. Application of Information Tecnology for Mass Customization in the Hosing Construction Industry in Korea. Automation in Construction, v. 17, p. 831-838, 2008.

SILVEIRA, G. D.; BORENSTEIN, D.; FOGLIATTO, F. S. Mass Customization: literature review and research directions. International Journal of Production Economics, v. 72, n. 1, p. 1-13, 2001.

SQUIRE, B. et al. The Impact of Mass Customization on Manufacturing Trade-Offs. Production and Operations Management, v. 15, n. 1, p. 10-21, 2006.

TIEN, J. M. Data Mining Requirements for Customized Goods and Services. International Journal of Information Technology \& Decision Making, v. 5, n. 4, p. 683-698, 2006. 
TIEN, J. M. Manufacturing and Services: from mass productions to mass customization. Journal of Systems Science and Systems Engineering, v. 20, n. 2, p. 129-154, 2011.

TIEN, J. M; KRISHNAMURTHY, A.; YASAR, A; Towards Real-Time Customized Management of supply and demand chains. Journal of Systems Science and Systems Engineering, v. 13, n. 3, p. 257-278, 2004.

TILlMANN, P. A.; FORMOSO, C. T. Táticas de Customização em Massa Para a Produção

Habitacional Brasileira: um estudo de caso. In:

ENCONTRO NACIONAL DE TECNOLOGIA

DO AMBIENTE CONSTRUÍDO, 12., Fortaleza, 2008. Anais... Fortaleza: ENTAC, 2008.
WIKNER, J.; RUDBERG, M. Integration Production and Engineering Perspectives on the Customer Order Decoupling Point. International Journal of Operations \& Production Management, v. 25, n. 7, p. 623-641, 2005.

YIN, R. K. Estudo de Caso: planejamento e métodos. 3. ed. Porto Alegre: Bookman, 2005.

\section{Agradecimentos}

Agradecemos a CAPES pela concessão de Bolsa para realização do Mestrado, e a COHAB_LB pela colaboração no desenvolvimento da pesquisa.

Jaqueline Taube

Centro Tecnológico | Universidade do Oeste de Santa Catarina | Rua Oiapoc, 211, Campus São Miguel do Oeste, Agostini | São Miguel do Oeste - SC - Brasil | CEP 89900-000 | Tel.: (49) 3631-1080 | E-mail: jaquetaubearq@gmail.com

Ercília Hitomi Hirota

Departamento de Construção Civil | Universidade Estadual de Londrina | Rodovia Celso Garcia Cid, Km 380 s/n, Campus Universitário | Londrina - PR - Brasil | CEP 86057-970 | Tel.: (43) 3371-4460 | E-mail: ercilia@uel.br

\section{Revista Ambiente Construído}

Associação Nacional de Tecnologia do Ambiente Construído

Av. Osvaldo Aranha, $99-3^{\circ}$ andar, Centro

Porto Alegre - RS - Brasil

CEP 90035-190

Telefone: +55 (51) 3308-4084

Fax: +55 (51) 3308-4054

www.seer.ufrgs.br/ambienteconstruido

E-mail: ambienteconstruido@ufrgs.br 